\title{
Review on Chronic Obstructive Pulmonary Disease Prediction System using Wheeze and Crackle.
}

This paper was downloaded from TechRxiv (https://www.techrxiv.org).

LICENSE

CC BY 4.0

SUBMISSION DATE / POSTED DATE

$18-06-2021$ / 22-06-2021

CITATION

De Alwis, Niwarthana (2021): Review on Chronic Obstructive Pulmonary Disease Prediction System using Wheeze and Crackle.. TechRxiv. Preprint. https://doi.org/10.36227/techrxiv.14810127.v1

$\mathrm{DOI}$

10.36227/techrxiv.14810127.v1 


\title{
Review on Chronic Obstructive Pulmonary Disease Prediction System using Wheeze and Crackle.
}

\author{
${ }^{1}$ Niwarthana Nihathamani De Alwis,University of Westminster, New Cavendish Street, London,UK \\ ${ }^{2}$ Achala Chathuranga Aponso, Informatics Institute of Technology,Colombo 6, Sri Lanka \\ nihathamanidealwis96@gmail.com
}

\begin{abstract}
As of today, Chronic Obstructive Pulmonary Disease (COPD) is a common lung disease in the community. Getting an accurate diagnosis is the most important step in treating a patient, allowing doctors to find the best treatment for their condition. Luckily, in today's World, Machine learning is making healthcare smarter. It's a complex process that requires human effort and time. The paper presents a better application design to identify this disease easily. The main aim is to predict that COPD patients can be performed with high accuracy according to respiratory sound reports. The latest technology will be used to achieve this goal through deep learning and Audio/speech processing. With this implementation of this research, it is hoped to make an accurate prediction of COPD and make it readily available to the patient.
\end{abstract}

Keywords: Chronic obstructive pulmonary disease, Artificial Intelligence, Machine learning, deep learning, Audio/speech processing.

\section{Introduction}

Anyone who has struggled to breathe knows how important it is even for a short time. The World Health Organization (WHO) has said $64 \%$ of million people suffer from chronic obstructive pulmonary disease (COPD). Every year more than three million people die from chronic obstructive pulmonary disease (COPD), which accounts for about $6 \%$ of deaths worldwide [1]. About $90 \%$ live in low- and middle-income countries. The World Health Organization estimates that by 2030 it will be the $3 \mathrm{rd}$ main cause of death worldwide and is expected to exceed $30 \%$ in the next 10 years [2].

Chronic obstructive pulmonary disease (COPD) is a lung disease. This is chronic obstruction of the airways in the lungs that interferes with normal breathing and but cannot be reversed. The terms 'chronic bronchitis' and 'emphysema' are no longer used and are now included in the diagnosis of COPD [2]. This is not a single disease. An application used to describe chronic lung disease. If the most common are chronic obstructive pulmonary disease, asthma, pulmonary hypertension, and occupational lung diseases.

\subsection{Signs and Symptoms}

These disease signs and symptoms are associated with emphysema, chronic bronchitis, and Reactive airways disease. Among the main signs and symptoms is difficulty breathing [3]. At first, these symptoms show a low level, but cough and shortness of breath are initial signs. A patient may experience chest tightness and wheeze. Also, COPD patients show severe symptoms, such as acute exacerbations. Some people consider this to be a common cold at the beginning of COPD, so the result is worse. Then symptoms include occasional shortness of breath, occasional cough, and sore throat. As a result, daily routines begin to change [4].

This has a severe effect on the lungs as the symptoms gradually worsen. Then the suffocation becomes too loud. It often manifests itself in situations such as walking and exercise. Every day I will start to suffer from colds, flu, and other respiratory ailments. Later symptoms of COPD include fatigue, swelling of the ankle or leg, and weight loss. As the oxygen level in the blood decreases, human nails and lips begin to change color.it also shows symptoms like dizziness when you do something fast [4]. 


\subsection{Diagnosis}

COPD diagnosis is made based on the symptoms, physical examination, and result of the diagnosis and there is no single test for this. It is imperative to see a doctor about this. Here the doctor uses a stethoscope to listen to the lungs as they breathe. Also, inquiries about medical history. That is smoking and related information, family history about this disease, asthma, or other respiratory diseases. Finally based on these facts the doctor conducts an examination. It's called Spirometry. This is an indeterminate test performed on the function of the lungs. There a tube linked to the spirometer is instructed to take a deep breath, and a chest x-ray or CT scan is taken. They can also take a blood sample from arteries [4]. Accordingly, the diagnosis is mainly made through Auscultation. Therefore, in this research, we make a diagnosis using Auscultation sound.

\subsection{Auscultation}

Respiratory sound plays a very important role in the diagnosis of respiratory system-related diseases. Respiratory sounds are sounds made by the respiratory system. They are heard by mucus. Respiratory sounds made of mucus may be Normal or abnormal sounds. Abnormal noise can be a major symptom of several serious respiratory illnesses, such as pneumonia, asthma, and COPD.

\section{Normal respiratory sound}

It is classified into several types according to the place where the normal respiratory sound is generated. That is, a Normal vesicular sound is heard in the Auscultation that performs the lungs area. It is easy to hear during inspiration, but this is heard at the beginning of the expiration. Broncho vesicular sound is heard between the scapulae in the back of the chest and the middle of the front chest. Bronchial sound is heard through the large airways in the chest, near the second and third intercostal area. Tracheal sound takes on a loud rough nature and can be heard when choking. And Mouth sound is produced by the central airway and is the sound of breathing through the mouth due to the turbulent air beneath the glutes [5].

\section{Abnormal respiratory sound}

The sounds that indicate a problem in the lungs are abnormal. This includes things that are not usually heard in the area where a sound should be heard, such as slowing down, changing, or hearing from areas where a sound can never be heard [6].

\section{Adventitious sound}

These are hyperventilation sounds. That is additional breathing sounds that are different from normal breathing sounds. This includes several adventitious lungs sounds but here we focus on wheeze and crackle.

\section{Wheeze}

This is a continuous adventitious sound. According to acoustics, it is a periodic waveform with a frequency of more than $100 \mathrm{hz}$ and $100 \mathrm{~ms}$. therefore at least then sequential vibrations are included. For these reasons, this is associated with an airway obstruction disruption. When a wheeze has a single frequency, it is classified as monophonic, and it has multiple frequencies it is classified as polyphonic. A wheeze can be situated at the site of an anatomic hindrance. Then the frequency is in the range of $100-2500 \mathrm{~Hz}$ [7].

\section{Crackle}

This is an adventurous explosive and continuous sound. Crackle shows up during the inspiration phase. These are specific waveforms. It is characterized by the duration and location of the respiratory cycle. Depending on the duration, it can be classified as short term (fine) or long term(coarse). Those indicate a pathological process in the affecting of the lungs tissue or airways, which are the sounds of the lungs. The crackle duration is less than $20 \mathrm{~ms}$ and the frequency range is between $100-200 \mathrm{hz}$ [8]. 

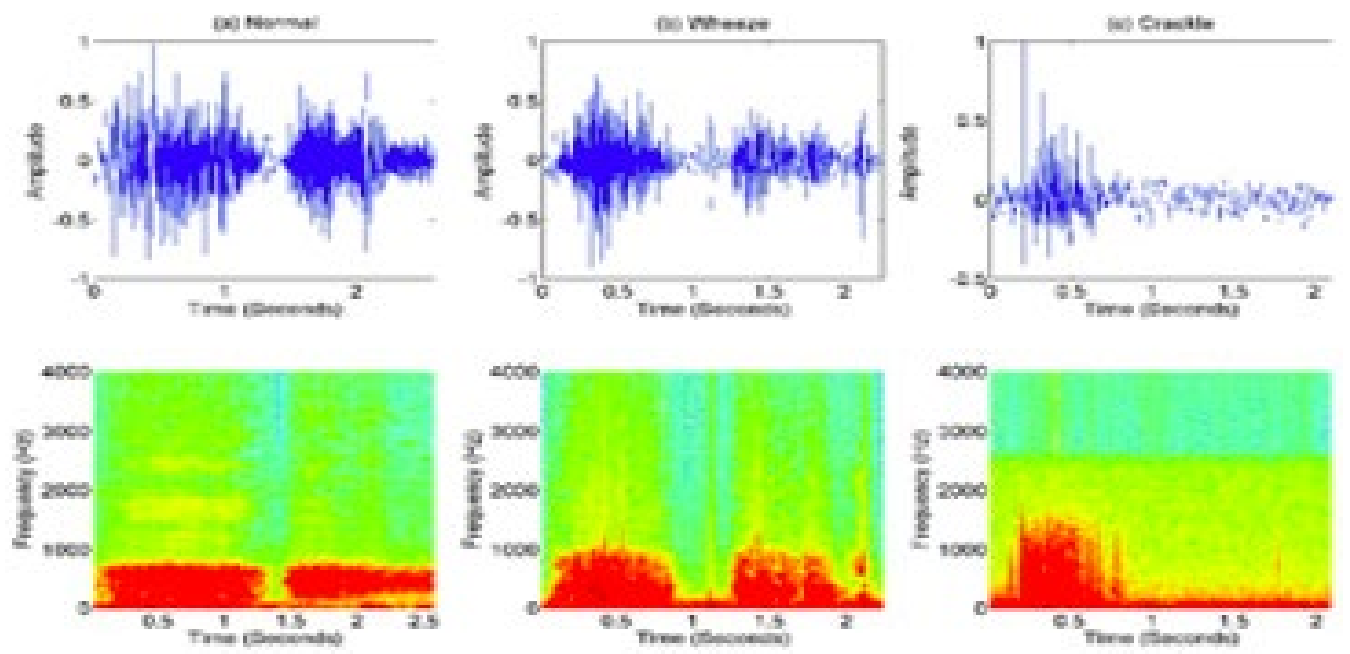

Fig. 1. Wheeze and Crackle

\section{Problem and Motivation}

The most effective way to control COPD is to diagnose the disease early and then seek appropriate medical treatment. Specially in a country like Sri Lanka, people may have to stay in the queue and suffer just to diagnose the disease. Therefore, a non-invasive, rapid, cost-effective, and easy procedure should be found to assess the condition of the patient's lungs. It should have the ability to easily teach even trained and untrained people. Here the experience of the doctor and the intensity of the ears are very important for the diagnosis. They have to get rid of the environmental noise and get the right lung sound. Therefore, we need to get the right diagnosis. In this research, we are trying to design and implement a system that can influence the diagnosis of COPD in a matter of seconds using modern technologies such as Artificial Intelligence (AI), Deep Learning (DP), and Convolutional Neural Networks (CNNs).

"The world health organization (WHO) has identified COPD as a major global unhealthy health requirement. This is a disease that affects one-tenth of the world's population. Within ten years COPD is expected to become the 3rd leading cause of death" [9]. The previous diagnosis has been identified as a major solution to disease control. This research has the opportunity to diagnose the disease at an early stage and implement solutions to make the system more efficient. This will save many lives by helping doctors and staff. This project enables the community to support them by enhancing and activating medical research with advancing technology.

\section{Existing Systems}

At present, researchers are very interested in the field of information technology and medicine. Accordingly, let us look at the research related to the project. 
"Method for automatic detection of wheezing in lung sounds (2009)" is a research project done by R.J. Riella, P. Nohama, and J.M. Maia [10]. This project describes a method of identifying wheeze in the digital record of lung sounds. This project focuses on two ways. It is spectral information extraction of the respiratory cycle and user feedback. Here the respiratory cycle is pre-set to normalize spectrally. then Performs spectroscopic calculation. After various procedures, spectral feedback is calculated from the spectrum and stored as an array to generate more compressed data for automatic detection. A multilayer perceptron artificial neural network is used to automatically detect magnitude values and related spectral values. The results show an accuracy of $84.82 \%$. It also presents the original recorded sounds and the postprocessed spectral image to help the user conclude [10].

This shows the success of the project detection of wheeze. But there are also flaws here.it is not possible to confirm whether a large number of errors can be computed in the detection of a sound, and it takes a long time to compute. It also shows that the time taken for the calculation can be reduced by refining the algorithm. Today this project can be improved with the advancement of technology [10].

"Prediction of Chronic Obstructive Pulmonary Disease (COPD) in Asthma Patients Using Electronic Medical Records (2009)" is a research project done by Blanca E. Himes, Isaac S Kohane, Scott T Weiss, and Marcos F Ramoni [11]. This project aims to find factors to control the focus of COPD growth among asthma patients. This uses a Bayesian network model of 8 comorbidity variables from the medical records. They use genetic information from adult asthma patients observed over five years from 1988 to 1998, and electronic medical report information from a partner's healthcare system. The information obtained from it predicts COPD in a group of independent patients and is calculated as "Area Under the Receiver Operating Characteristics curve" (AUROC) [11].

COPD growth is higher in patients with asthma, which has led to the use of various chemical methods, including medical records and smoking. Then, the main task is to create a predictive model based on comorbidity and genetic information from asthma patient data. The result shows an accuracy of $83.3 \%$ in this project. It has an AUROC value of 0.83. therefore, they point out that data from electronic medical records can make a successful prediction. But it also shows that the system should include a variable model to find out how COPD progression changes as asthma patients get older. And the doctors should be well aware of this before doing the test. This knowledge is limited to a few doctors. Due to that reason when inexperienced doctors test it the results face problems [11].

"Signal Domain in Respiratory Sound Analysis" (2015) is a research project done by Achmand Rizal, Risanuri Hidayat and Hanung Adi Nugroho [12]. This research aims to use digital signal technology to automatically detect lung sound and draw conclusions based on their view. Here they mean the detection of abnormalities in the lungs and respiratory organs using lung sound. Mainly classifies and discusses lung sound using signal processing. Then interprets lung sounds within each of those domains. Lung sounds are initially assessed manually according to the doctor's specificity.

The researchers also developed a classification of sensors, datasets, and sounds extraction techniques used in previous projects. They prove that it is more practical to store lung sound as key data for diagnosis. Eventually, they also point out that the processing of lung sound signals achieves high accuracy. But they could not find out what is the best domain for signal processing. This cannot be diagnosed and obtained directly so there is a need to improve this until then [12].

"An artificial intelligence approach to early predict symptom-based exacerbations of COPD " (2018) is research done by Miguel Angel Fernandez, Daniel Sanchez Morillo, and Antonio Leon Jimenez [13]. This project aims to create a COPD automation system based on acute exacerbation symptoms by recording respiratory sounds. For this purpose, selected patients are given tele monitored within 6 months and recorded. This is done using parts taken from home-recorded respiratory sounds with a breathing sensor. It's ad hoc created. The Decision tree forest classifier is used to classify them automatically according to their symptoms. 
It shows that they were able to predict average COPD exacerbation within the initial 4.4 days. They point out that $75.8 \%$ of the final results were reported as exacerbation Also $87.5 \%$ of the final results were unreported as an exacerbation. But they predict that the result will be significant support for machine learning to detect COPD exacerbation early. It is hoped that this will help to create a stable algorithm for electronic sensors [13]. But there is a possibility of errors in the results when the respiratory sounds are not recorded correctly.

"Respiratory sound analysis in the era of evidence-based medicine and the world of medicine 2" (2018) is a research project done by E Andres, R Gass, A Charioux, C Brandt, and A Hentzler [14]. This research describes respiratory sound analysis related to the state of the art, ongoing research, and scientific publication. They have conducted an independent study on how to analyze respiratory sounds during the period up to 2018. This has been done using research done by various researchers for respiratory sound analysis. For that, a very clear study of the current research on various topics can be seen in this project. They also point out that the difference between a normal lung sound and an abnormal sound (like wheeze and crackle) is very important to make an accurate diagnosis. Also, there is a possibility that problems may arise in making such a diagnosis using a stethoscope.

They explain that these shortcomings could be avoided by electrical malfunctions and automatic classification of recorded lung sounds. Currently, artificial neural networks, genetic algorithms, and fuzzy system research are the most accurate research described in this journal. In this, it has tested the algorithms, but no study of spectrum and signal analysis techniques conducted [14].

"Evaluation of features for classification of wheezes and normal respiratory sounds" (2019) is research done by Renard Xaviero Adhi Pramono, Syed Anas Itiaz, Esther Rodriguez [15]. The main objective of this project is to present a comprehensive study of the variability of various components for the automatic detection of breathing problems. Because COPD and Asthma cannot be cured, the disease can be controlled by early detection and monitoring of symptoms. To do this, they use time, spectra, wavelengths, and cepstral features. Information with 105 individual characteristics is evaluated here.

These classifications use a Linear Classifier to determine the single features. Although this is a simple classification, complex classification such as artificial neural networks (ANN) and Support Vector Machines (SVM) also used Logistic Regression to determine multiple features. then it can be used to increase performance. wheeze detection shows more accuracy in individual traits. Tonality index and Mel frequency cepstral coefficients (MFCC) value are higher than other features. That is the Tonality index had a result of $87.95 \%$, which was found to be the lowest p-value in the rating test. Also, they point out that the use of multiple features is sometimes highly valued, and also use of algorithmic designs to detect automatic wheeze is very useful for researchers. here concludes the study by analyzing the different trades between the requirements for calculating the accuracy of different components. [15].

\section{Comparison of existing system project}

\begin{tabular}{|l|l|l|l|}
\hline Research & Aim & Improvement & Limitation \\
\hline$[10]$ & $\begin{array}{l}\text { Using an image processor- } \\
\text { based spectrum to } \\
\text { automatically detect lung } \\
\text { sound. }\end{array}$ & $\begin{array}{l}\text { The spectrogram is used } \\
\text { to obtain information } \\
\text { about the respiratory } \\
\text { cycle. }\end{array}$ & scalability issues. \\
\hline$[11]$ & $\begin{array}{l}\text { Find factors to control the } \\
\text { focus of COPD growth } \\
\text { among asthma patients }\end{array}$ & $\begin{array}{l}\text { Used machine learning } \\
\text { and algorithms for more } \\
\text { accuracy. }\end{array}$ & $\begin{array}{l}\text { project data knowledge is } \\
\text { spread only among a } \\
\text { limited number } \\
\text { people. }\end{array}$ \\
\hline
\end{tabular}




\begin{tabular}{|c|c|c|c|}
\hline [12] & $\begin{array}{lrr}\text { Automatic lung } & \text { sound } \\
\text { detection } & \text { using } & \text { digital } \\
\text { signal processing. } & \end{array}$ & $\begin{array}{l}\text { Discussing the different } \\
\text { aspects of digital signal } \\
\text { processing in lung } \\
\text { sounds. }\end{array}$ & $\begin{array}{l}\text { Supported only selected } \\
\text { classifications. }\end{array}$ \\
\hline [13] & $\begin{array}{l}\text { To create a COPD } \\
\text { automation system based } \\
\text { on acute exacerbation } \\
\text { symptoms. }\end{array}$ & $\begin{array}{l}\text { Used algorithms for } \\
\text { more efficiency. }\end{array}$ & $\begin{array}{l}\text { Creating a research- } \\
\text { specific electronic device } \\
\text { using sensors. }\end{array}$ \\
\hline [14] & $\begin{array}{l}\text { Identify projects with } \\
\text { higher accuracy through } \\
\text { sound repository analysis } \\
\text { from existing research. }\end{array}$ & $\begin{array}{l}\text { Explains the use of } \\
\text { algorithms in respiratory } \\
\text { sound analysis very well. }\end{array}$ & NA \\
\hline [15] & $\begin{array}{l}\text { to present the variability of } \\
\text { various components for the } \\
\text { automatic detection of } \\
\text { breathing problems }\end{array}$ & $\begin{array}{l}\text { The classification is done } \\
\text { using various elements } \\
\text { for automatic } \\
\text { identification. }\end{array}$ & $\begin{array}{l}\text { Performed based on } \\
\text { selected features (time, } \\
\text { spectra, wavelengths, } \\
\text { and cepstral features) }\end{array}$ \\
\hline
\end{tabular}

Table 1. Comparison of existing system

\section{STUDY SETUP}

In the previous few years, Artificial Intelligence (AI) has been a subject of acute concern. AI, Machine learning, and deep learning have become much talked about topics. Artificial Intelligence (AI) "is the ability of a computer or digital machine to achieve a function that traditionally requires human intelligence" [16].

First, we can check existing system project algorithms comparison.

\subsection{Comparison of algorithm section method}

\begin{tabular}{|l|l|l|}
\hline Algorithms & Advantages & Disadvantages \\
\hline $\begin{array}{l}\text { Linear } \\
\text { regression }\end{array}$ & $\begin{array}{l}\text { Have suitable and record-breaking } \\
\text { performances with smaller datasets. Its } \\
\text { output can be interpreted as a probability } \\
{[20] .}\end{array}$ & $\begin{array}{l}\text { Data assumptions are needed to have } \\
\text { complied. It can be only provided } \\
\text { linear solutions only [20]. }\end{array}$ \\
\hline Decision Tree & $\begin{array}{l}\text { It has performance to handle categorical } \\
\text { features. There are some parameters to } \\
\text { tune. Those play well in datasets even with } \\
\text { the largest number of attributes [17]. }\end{array}$ & $\begin{array}{l}\text { Interpretability of ensembles can be } \\
\text { questioned [17]. }\end{array}$ \\
\hline
\end{tabular}




\begin{tabular}{|l|l|l|}
\hline $\begin{array}{l}\text { Support Vector } \\
\text { Machines }\end{array}$ & $\begin{array}{l}\text { It helps us to have a non-linear solution. } \\
\text { And also, it is so easy to use complexity } \\
\text { [17]. }\end{array}$ & $\begin{array}{l}\text { To have a good performance, they } \\
\text { require knowledge about the kernel } \\
\text { employed [17]. }\end{array}$ \\
\hline $\begin{array}{l}\text { Bayesian } \\
\text { Network }\end{array}$ & $\begin{array}{l}\text { Bayesian Network makes the any type of } \\
\text { calculation process easier. Also, have a } \\
\text { better speed and accuracy for huge } \\
\text { attributes [18]. }\end{array}$ & $\begin{array}{l}\text { It does not give accurate results for } \\
\text { some cases where there exists } \\
\text { dependency among variables [18]. }\end{array}$ \\
\hline $\begin{array}{l}\text { Genetic } \\
\text { Algorithm }\end{array}$ & $\begin{array}{l}\text { The convergence accuracy is faster, and } \\
\text { the versatility is stronger [19]. }\end{array}$ & $\begin{array}{l}\text { It is most complex, easy to fail into } \\
\text { premature convergence, It always } \\
\text { depends on the initial population } \\
\text { [19]. }\end{array}$ \\
\hline ANN & $\begin{array}{l}\text { Prophetic power and also, having the } \\
\text { ability to discover non-linear relationships } \\
\text { too [20]. }\end{array}$ & $\begin{array}{l}\text { Have a minimum interpretable, weak } \\
\text { scalability for handling large volume } \\
\text { of data sets [20]. }\end{array}$ \\
\hline $\begin{array}{l}\text { Recursive } \\
\text { Neural } \\
\text { Networks }\end{array}$ & $\begin{array}{l}\text { Support time series data and possess } \\
\text { internal memory for storing information } \\
\text { data [21]. }\end{array}$ & $\begin{array}{l}\text { Only able to store one layer of data } \\
\text { and information [21]. }\end{array}$ \\
\hline CNN & Feature extract and learn-full [22]. & $\begin{array}{l}\text { Need a huge amount of data for } \\
\text { training [22]. }\end{array}$ \\
\hline
\end{tabular}

Table 2. Comparison of algorithms

After studying all the previous projects and related technologies, we have to select a suitable model for our project. Here they basically advised us on machine learning and deep learning algorithms. Accordingly let us consider the following quantitative methods to select a suitable model form these two types.

\begin{tabular}{|l|l|}
\hline \multicolumn{2}{|c|}{ Machine Learning How it works } \\
\hline \multicolumn{2}{|c|}{ Deep Learning } \\
\hline $\begin{array}{l}\text { There are various types of automated algorithms } \\
\text { that learn to model functions and predict future } \\
\text { actions from predicted data. }\end{array}$ & $\begin{array}{l}\text { By the use of neural networks, that passes data } \\
\text { through some processing layers to interpret } \\
\text { data and features and relations. }\end{array}$ \\
\hline \multicolumn{2}{|c|}{ Optimal Data Volumes } \\
\hline $\begin{array}{l}\text { All the algorithms are detected by the way of data } \\
\text { analysts to examine specific clarifications in data } \\
\text { sets. }\end{array}$ & $\begin{array}{l}\text { Algorithms which use here are largely self- } \\
\text { directed on data analysis after they are put into } \\
\text { production. }\end{array}$ \\
\hline \multicolumn{2}{|c|}{ Output } \\
\hline Thousands of data points; low data points & $\begin{array}{l}\text { Big data: millions of data points; higher data } \\
\text { points }\end{array}$ \\
\hline \multicolumn{2}{|c|}{$\begin{array}{l}\text { Anything forms numerical values to free form } \\
\text { elements, as an example, free text and sounds }\end{array}$} \\
\hline Numerical value, like rankings or score
\end{tabular}




\begin{tabular}{|l|l|}
\hline \multicolumn{2}{|c|}{ Accuracy } \\
\hline Gives lesser accuracy to process & Provides a higher accuracy to process \\
\hline
\end{tabular}

Table 3. Comparison of selected algorithms

After considering these factors, it seems appropriate to select a Deep Learning Algorithm to get the best results for the project.

In the end according to all the research information wheeze and crackle can be used to diagnose in COPD, and we try to predict this diagnosis using deep learning algorithms and signal processing technology.

\subsection{Signal Processing}

We identified a few projects that used signal processing for respiratory sound analysis.

\section{Fourier Transform}

Often the signal is non-static and changes with time. Then the essential conversion of a signal from the time domain to the persistence domain is called Fourier transformation. Where it tests the existence and strength of different frequencies. Causes such as modulation, signal filtration, and signal processing by the most appropriate methods are considered advantages here. The calculation is done by the Discrete Fourier Transform (DFT) [22].

\section{Power spectral Density}

Power spectral Density describes the form of a certain frequency variable. That is strong or weak. PSD represents the power of a signal or time sequence at various frequencies. This is the Fourier transform of the automated connection function that provides conversion from the time domain to the persistence domain [23].

\section{Mel Spectrogram}

We explained in the previous sections that a transformation that takes place over a period of time is treated as a signal, and that it consists of several signal frequency sound waves in a sound signal. We also call it the Fourier transform. The end result is spectrum. Then the frequency of a signal is described by the content Fast Fourier transform (FFT). Here the FFT calculation is done overlapping the window sections. It's obtained through spectrogram. Spectrograms visually represent the sound or amplitude of a signal. Previous studies show that human frequencies do not take on a linear scale. In 1937 Newmann, Volkmann and Stevens introduced a pitch unit that the same distances as the pitch, is the same to the distances to the listener. This scale is called Mel Spectrogram. The Mel spectrogram then portrays the acoustic time frequency of a sound. This is the result of converting spectral values to Mel scale. That is the spectrum that converts frequencies to the Mel scale is called the Mel Spectrogram [24].

\section{Mel- frequency Cepstral Coefficients (MFCC)}

MFCC operates on the human terminal auditory system. Here human cognition about frequency content uses this for speech signals. But here the sound liner scale is not followed. Every tone with its actual frequency is measured in Hz. Furthermore, subject matter pitch is measured by the Mel scale. This scale is linear frequency less than $1000 \mathrm{~Hz}$ and greater than $1 \mathrm{kHz}$ logarithmic distance. This is calculated from individual windows characterized by a huge variance. However, this signal processing is widely used for speech recognition and audio processing [25]. 


\section{Conclusions}

After studying the various projects, it does not show an accurate, easy diagnostic method. For this reason, it would be more appropriate to diagnose COPD by wheeze and crackling after considering the finding of the projects. It also looks to design a more efficient application using new technology. It was also discussed that diagnosing diseases using respiratory audio sound will help in quick diagnosis. Further, the existing system used Bayesian Network, genetic algorithms, Digital signal processing techniques, Linear regression for diagnosis of COPD. Since the Algorithms for diagnosis diseases using respiratory sounds have already been outdated. Because the latest technologies have been introduced today. Accordingly, the discussion on how to make predictions using the latest algorithms was done here. there it proved to be more appropriate to use a Deep Learning Algorithm for more efficient results. In this case, an appropriate method should be used for audio signal processing when the input data is obtained by respiratory sound. After discussing the audio signal processing methods, it seems that is more appropriate to use the Mel Spectrogram for this designed application. Considering all the factors, this application will enable COPD patients to get a diagnosis very quickly and seek treatment at an early stage.

\section{Acknowledgements}

The efforts are taken towards the completion of this project were extremely challenging and were a delightful experience. However, it would not have been possible without the assistance and the greater good support of the many people who worked with me. First, I would like to express my sincere gratitude and thanks to my supervisor for his support, guidance throughout the project. I would also like to thank my friends and senior students at the Informatics Institute of Technology. Finally, I would like to thank my parents for their love and support, without them, this would not have been possible.

\section{References}

1. Who. 2020. COPD: Definition: who.int

2. Who. 2020. Chronic Respiratory Diseases: who.int

3. Mosenifar, Z., 2020. Chronic Obstructive Pulmonary Disease (COPD): Practice Essentials, Background, Pathophysiology.

4. Prasad, B., 2019. Chronic Obstructive Pulmonary Disease (COPD).

5. Bowyer, S., Adhi Pramono, R. and Rodriguez, E., 2017. Automatic Adventitious Respiratory Sound Analysis: A Systematic Review.

6. Izbicki, G., Bohadana, A. and Kraman, S., 2014. Fundamentals Of Lung Auscultation | NEJM. New England Journal of Medicine.

7. E Andrès, A., 2018. Respiratory Sound Analysis In The Era Of Evidence-Based Medicine And The World Of Medicine 2.0 : PubMed Central (PMC).

8. Reichert, S., Gass, R., Brandt, C. and Andrès, E., 2008. Analysis Of Respiratory Sounds: State Of The Art: Journals.sagepub.com.

9. Fazleen, A. and Wilkinson, T., 2020. Early COPD: Current Evidence For Diagnosis And Management. SAGE Journals.

10. Riella, R., Nohama, P. and Maia, J., 2009. Method For Automatic Detection Of Wheezing In Lung Sounds.

11. Himes, B., Dai, Y., Kohane, I., Weiss, S. and Ramoni, M., 2009. Prediction Of Chronic Obstructive Pulmonary Disease (COPD) In Asthma Patients Using Electronic Medical Records.

12. Rizal, A., Hidayat, R. and Adi Nugroho, H., 2015. [online] Thescipub.com.

13. Fernandez, M., Sanchez, D. and Leon, A., 2018. An Artificial Intelligence Approach To Early Predict SymptomBased Exacerbations Of COPD.

14. E Andrès, A., 2018. Respiratory Sound Analysis In The Era Of Evidence-Based Medicine And The World Of Medicine 2.0.

15. Pramono, R., Imtiaz, S. and Rodriguez-Villegas, E., 2019. Evaluation Of Features For Classification Of Wheezes And Normal Respiratory Sounds.

16. Bali, J. and Bali, O., 2019. Artificial Intelligence Applications In Medicine: A Rapid Overview Of Current Paradigms. 
17. S. V Nesterov, S. A Kajander, O. Martinez Manzanera, L. Juárez-Orozco and J. M Knuuti,2018., The machine learning horizon in cardiac hybrid imaging, researchgate.net.

18. D. Tomar, 2013., A survey on Data Mining approaches for Healthcare, researchgate.net.

19. L. Yang, X. Yang, Y. Wu and X. Liu, 2018, Applied Research on Distributed Generation Optimal Allocation Based on Improved Estimation of Distribution Algorithm, researchgate.net.

20. T. Cristóbal, G. Padrón, A. Quesada-Arencibia, F. Alayón, G. de Blasio and C. García, 2019., Bus Travel Time Prediction Model Based on Profile Similarity, researchgate.net.

21. L. Chian et al. 2017., SEGMENTATION-FREE LICENSE PLATE RECOGNITION USING DEEP LEARNING SOO CHING PAU A project report submitted in partial fulfilment of the requirements for the award of Bachelor of Science (Hons.) Software Engineering Semanticscholar.org,.

22. Zhu, Q., Wang, Y. and Shen, G., 2011. Comparison And Application Of Time-Frequency Analysis Methods For Nonstationary Signal Processing.

23. Lee, E. and Eun, H., 2016. Structural Damage Detection By Power Spectral Density Estimation Using OutputOnly Measurement.

24. Stevens, S., Volkmann, J. and Newman, E., 2005. A Scale For The Measurement Of The Psychological Magnitude Pitch.

25. Tiwari, 2010. MFCC and its applications in speaker recognition: International Journal on Emerging Techno 
